\title{
Intraparenchymal treatment with bone marrow mesenchymal stem cell-conditioned medium exerts neuroprotection following intracerebral hemorrhage
}

\author{
CHANGMENG CUI ${ }^{1}$, YING CUI ${ }^{2}$, JUNLING GAO $^{3,4}, \mathrm{RAN} \mathrm{LI}^{3,4}$, \\ XIAOHUA JIANG ${ }^{3,4}$, YANXIA TIAN ${ }^{3,4}$, KAIJIE WANG ${ }^{2}$ and JIANZHONG CUI ${ }^{1,2}$
}

${ }^{1}$ Department of Surgery, Hebei Medical University, Shijiazhuang, Hebei 050017; ${ }^{2}$ Department of Neurosurgery,

Tangshan Workers' Hospital; ${ }^{3}$ Department of Histology and Embryology, School of Basic Medical Science;

${ }^{4}$ Hebei Key Laboratory for Chronic Diseases, Tangshan Key Laboratory for Preclinical and Basic Research on

Chronic Diseases, North China University of Science and Technology, Tangshan, Hebei 063000, P.R. China

Received December 8, 2015; Accepted January 12, 2017

DOI: $10.3892 / \mathrm{mmr} .2017 .6223$

\begin{abstract}
Intracerebral hemorrhage (ICH) is a life-threatening type of stroke. Previous studies have reported that bone marrow mesenchymal stem cells (BMSCs) may exert beneficial effects on the treatment of $\mathrm{ICH}$. However, it remains unknown whether the neuroprotection exerted by BMSCs on $\mathrm{ICH}$ is due to the differentiation of BMSCs, or the trophic factors secreted into their conditioned medium (CM). In addition, growth-associated protein-43 (GAP-43) is a protein associated with neurite extension, which may be considered a prospective therapeutic target in the treatment of ICH. The present study investigated whether administration of BMSC-CM could be considered as an alternative to the established treatment of direct BMSC transplantation; in addition, the underlying mechanisms were evaluated. Neurological function tests, brain water content, reverse transcription -quantitative polymerase chain reaction and western blotting were used in present study. The current study indicated that the neuroprotective effects of BMSC implantation and BMSC-CM treatment are similar, and that both decrease the severity of post-ICH cerebral edema, as well as improving neurological functions. At the molecular
\end{abstract}

Correspondence to: Dr Jianzhong Cui, Department of Surgery, Hebei Medical University, 361 East Zhongshan Road, Shijiazhuang, Hebei 050017, P.R. China

E-mail: jzhcuidr2001@163.com

Abbreviations: ICH, intracerebral hemorrhage; GAP-43, growthassociated protein-43; CNS, central nervous system; BMSCs, bone marrow mesenchymal stem cells; CM, conditioned medium; mNSS, modified neurological severity score; DMSO, dimethyl sulfoxide; NSCs, neural stem cells; MSCs, mesenchymal stem cells; BDNF, brain-derived neurotrophic factor; NGF, nerve growth factor; VEGF, vascular endothelial growth factor

Key words: intracerebral hemorrhage, bone marrow mesenchymal stem cells, conditioned medium treatment, inflammation, GAP-43 level, treatment with BMSC-CM resulted in a marked elevation in the expression of GAP-43 and interleukin (IL)-10, in addition to a significant reduction in the expression levels of IL-1 $\beta$, tumor necrosis factor- $\alpha$ and IL-6. Following application of a phosphorylated-extracellular signal-regulated kinase (ERK1/2) inhibitor, PD98059, in a BMSC-CM rat model, the mRNA and protein expression levels of GAP-43 were significantly attenuated. Therefore, the findings of the present study demonstrated that treatment with BMSC-CM may be an alternative to direct BMSC transplantation in a rat model of ICH. The mechanism underlying BMSC-CM-mediated neuroprotection may be associated with anti-inflammatory effects, as well as activation of GAP-43 transcription and expression through ERK-1/2 phosphorylation. Therefore, the ERK-1/2-GAP-43 signaling pathway may be considered a potential novel application target of BMSC-CM for the treatment of neurological diseases.

\section{Introduction}

Stroke is the fourth major cause of morbidity and remains a leading challenge to public health (1). Intracerebral hemorrhage $(\mathrm{ICH})$ is a life-threatening type of stroke that affects 2 million individuals annually worldwide (2). The symptoms, which differ in accordance to the site and spectrum of brain damage, have the potential to be seriously disabling and may lead to impaired motor and sensorimotor functions, along with cognitive impairments (3). ICH is characterized by extravasation of blood into the brain parenchyma, leading to hematoma formation, concomitant brain edema and secondary brain damage (4). Previous studies regarding secondary brain damage have identified possible treatments that target brain edema, blood-brain barrier disruption, inflammation and the decomposition product of erythrocytes (5-7). However, despite considerable progress being made in animal models and preclinical research in recent years, there are currently no available therapeutic strategies in clinical practice for ICH (8).

Growth-associated protein-43 (GAP-43) is a protein associated with neurite growth during development and regeneration, 
which allows differentiating neurons to respond to extracellular signals of the central nervous system (CNS) (9). A previous study demonstrated that mice lacking this protein have a low survival rate during the early postnatal period (10). In human neuropathologies and their associated animal models, complex alterations in GAP-43 expression are frequently detected, suggesting axonal damage or attempts of regenerative axonal sprouting. A previous in vivo study demonstrated the requirement of GAP-43 in sustaining synaptic stability and accelerating the initiation of axonal regrowth (11). In particular, one of the most marked regenerative events following the onset of stroke is axonal sprouting in the penumbra, which is accompanied by high expression of GAP-43 (12-14). Therefore, it was hypothesized that GAP-43 may be a prospective therapeutic target for the treatment of $\mathrm{ICH}$.

Bone marrow mesenchymal stem cells (BMSCs) are multipotent cells with self-renewal capabilities, which are able to differentiate into various cell types, including adipocytes, osteoblasts, chondroblasts and neural cells (15-17). In addition, it has previously been indicated that BMSCs may modify the tissue microenvironment via secreting soluble factors to rejuvenate or reinstate diseased cells (18). Such factors secreted from BMSCs serve a considerable role in various respects of hematopoiesis and are collectively termed trophic factors (19). Therefore, BMSC-induced reinstating of dysfunctional tissues could result from secretion of these trophic factors and/or their differentiation. Previous studies have demonstrated that intraparenchymal transplantation of BMSCs can exert anti-inflammatory and regenerative effects, and ameliorate neurological deficits in numerous CNS diseases, including stroke (20-22). However, the mechanisms by which BMSC implantation provides neuroprotection under CNS pathological circumstances are not well understood. Since transplantation of stem cells is often associated with poor differentiation and survival rates, utilization of conditioned medium (CM), which includes trophic factors from stem cells, has emerged as an alternative method $(23,24)$. A recent study confirmed that $\mathrm{CM}$ from human umbilical cord MSCs has considerable potential for stroke treatment without requiring stem cell transplantation (25). Therefore, the application of stem cell-CM in place of direct transplantation of stem cells may overcome the restrictions of current cell-based therapies. However, it remains unknown whether the therapeutic action of BMSCs is due to their differentiation or the trophic factors secreted into their CM.

In the present study, BMSC-CM was tested as an alternative to direct BMSC transplantation. To investigate the effects of BMSC-CM, the alterations in expression of GAP-43, phosphorylated-extracellular signal-regulated kinase (p-ERK1/2), p-c-Jun $\mathrm{N}$-terminal kinase (JNK) and inflammatory factors [interleukin (IL)-1 $\beta$, tumor necrosis factor (TNF)- $\alpha$, IL-6 and IL-10] in the perihematomal tissues were assessed. The mechanisms underlying the effects of BMSC-CM were also examined, in order to identify whether it was associated with, at least in part, the secretion of neurotrophic factors that activate the ERK1/2 signaling pathway and thereby elevate GAP-43 expression.

\section{Materials and methods}

Animals. All rat experiments were performed in compliance with the guidelines of the National Institutes of Health
Guide (2011) for the Care and Use of Laboratory Animals (Bethesda, MD, USA). The present experimental procedures were approved by the Hebei Medical University Experimental Ethics Committee (Shijiazhuang, China). A total of 151 male Sprague-Dawley rats (8 weeks old; weight, 250-280 g) and 6 male Sprague-Dawley rats (3 weeks old; weight, 90-110 g) purchased from the Hebei United University Experimental Animal Center (Tangshan, China) were used in present study. Rats were anesthetized with $10 \%$ chloral hydrate $(100 \mathrm{mg} / \mathrm{kg}$, Bio-Rad Laboratories, Inc., Shanghai, China). All rats were maintained under a $12 \mathrm{~h} \mathrm{light} / 12 \mathrm{~h}$ dark cycle at $22-24^{\circ} \mathrm{C}$ with ad libitum access to food and water. Great efforts were made to minimize the number of animals used and alleviate animal suffering.

Primary culture of BMSCs. BMSCs were generated from 6 male Sprague-Dawley rats (3 weeks old; weight, 90-110 g). The medullary cavities of rat femurs were flushed with Dulbecco's modified Eagle's medium (DMEM; Gibco; Thermo Fisher Scientific, Inc., Waltham, MA, USA) to collect fresh bone marrow cells. Cells were centrifuged at 1,000 x $g$ for $5 \mathrm{~min}$. Purified cells were dispersed in cell culture flasks at $37^{\circ} \mathrm{C}$ (Corning Life Sciences, Tewksbury, MA, USA), and were cultured in DMEM supplemented with $10 \%$ fetal bovine serum (Gibco; Thermo Fisher Scientific, Inc.), $100 \mathrm{U} / \mathrm{ml}$ penicillin and $100 \mu \mathrm{g} / \mathrm{ml}$ streptomycin (Sigma-Aldrich; Merck KGaA, Darmstadt, Germany) at $37^{\circ} \mathrm{C}$ in an atmosphere containing $5 \% \mathrm{CO}_{2}$. After $48 \mathrm{~h}$, non-adherent cells were removed and fresh media was added. Media was replaced every 3 days. After primary culturing, the cells were subcultured at a density of $1 \times 10^{4}$ cells $/ \mathrm{cm}^{2}$ and used for further experimentation after three passages.

Preparation of BMSC-CM. BMSCs from three passages were cultured in culture flasks. When cells reached $80 \%$ confluence, they were cultured in $10 \mathrm{ml}$ serum-free DMEM/F12. CM from BMSCs was collected after 1 day and centrifuged at $560 \mathrm{x} g$ for $10 \mathrm{~min}$ at $37^{\circ} \mathrm{C}$. After the supernatants were re-centrifuged at $5,040 \mathrm{x} \mathrm{g}$ for $5 \mathrm{~min}$ at $37^{\circ} \mathrm{C}$, the $\mathrm{CM}$ was collected and used for further experimentation.

ICH model. The rat ICH model was established as described by a previous study (26). Rats were anesthetized with an intraperitoneal injection of chloral hydrate $(100 \mathrm{mg} / \mathrm{kg}$ body weight) and placed on stereotaxic apparatus. A hole was drilled into the skull, and a needle was inserted into the right basal ganglia under stereotactic guidance (coordinates: $0.2 \mathrm{~mm}$ anterior, $5.5 \mathrm{~mm}$ ventral, and $3.5 \mathrm{~mm}$ lateral to the midline). A total of $100 \mu$ l autologous arterial blood was infused slowly (5 $\mu \mathrm{l} / \mathrm{min}$ ) with a microinfusion pump. Following the completion of the infusion, the needle was left in place for $20 \mathrm{~min}$ prior to withdrawal. Control rats received an infusion of $100 \mu \mathrm{l}$ normal saline. Bone wax was placed around the burr hole and all procedures were conducted under aseptic conditions to avoid infection.

Experiment groups. Three experiments were performed as follows. Experiment 1 was conducted to determine the time course of brain water content and functional recovery. A total of 101 rats were randomly assigned to four groups: The normal 
control group ( $\mathrm{n}=8$, Sham); the ICH model group $(\mathrm{n}=31$, ICH); the BMSC transplantation (10 $\mu 1$, tail vein injection immediately post-ICH) after $\mathrm{ICH}$ group $(\mathrm{n}=31$, BMSCs); and the BMSC-CM treatment (10 $\mu$ l, tail vein injection immediately post-IC) after ICH group $(\mathrm{n}=31, \mathrm{CM})$. A forelimb-placing test, corner turn test and modified neurological severity score (mNSS) evaluation were used to detect motor and sensorimotor functions 1 day prior to and 1-7 days after surgery. The time course variation of brain edema was evaluated by analysis of brain water content 1-7 days following ICH. Cognitive impairment of the rats was evaluated by Morris water maze test 7-10 days following ICH.

Experiment 2 was conducted to identify the neuroprotective mechanism of BMSC-CM. A total of 30 rats were randomly divided into three groups: The sham-operated group $(n=10$, Sham); the ICH model group $(n=10, I C H)$; and the BMSC-CM treatment after ICH group $(n=10, C M)$. A total of 4 days after ICH or sham operation, reverse transcription-polymerase chain reaction (RT-PCR) and western blot analysis were used to detect the expression levels of axonal regrowth-associated protein GAP-43, p-ERK, p-JNK and inflammatory cytokines.

Experiment 3 was conducted to determine the regulation of GAP-43 transcription and expression following BMSC-CM treatment. A total of $20 \mathrm{ICH}$ rats treated with BMSC-CM were randomly divided into two groups: The vehicle group, which received an equal volume of 5\% dimethyl sulfoxide (DMSO; $\mathrm{n}=10$, vehicle); and the PD98059 group, which received $5 \mu \mathrm{g}$ p-ERK1/2 inhibitor PD98059 (Bio-Rad Laboratories, Inc.) dissolved in 5\% DMSO ( $\mathrm{n}=10$, PD98059). The drug injection volume was $20 \mu \mathrm{l}$ followed by a flush with $10 \mu \mathrm{l} 0.9 \%$ saline via intrathecal injection 1 day after ICH.

Neurological function tests. Forelimb-placing test, corner turn test and an mNSS evaluation were performed prior to surgery and 1-7 days after ICH by an investigator who was blinded to the experimental groups. A total of 15 rats (5 per group) were used for these tests.

Forelimb placing was scored using a vibrissae-elicited forelimb-placing test. Independent testing of each forelimb was induced by brushing the vibrissae ipsilateral to that forelimb on the edge of a tabletop. Intact animals placed the forelimb quickly onto the countertop. This task was repeated 10 times and the percentage of successful placing responses was determined.

In the corner turn test, rats were allowed to proceed into a $30^{\circ}$ corner. To exit the corner, the rat could turn either left or right, and the direction was recorded. This task was repeated 10 times.

The mNSS evaluation is a composite of motor, sensory, balance and reflex tests. Neurological function was graded on a scale of 0-18 (0, normal score; 18, maximal deficit score) with one point awarded for the exhibition of specific abnormal behavior or for lack of a tested reflex. A greater impairment of normal function results in a higher score.

Evaluation of brain edema. Brain edema was evaluated by analysis of brain water content, as previously described (27). A total of 66 rats were used to evaluate brain edema (Sham, 3; $\mathrm{ICH}, 21$; BMSCs, 21; CM, 21). The percentage of water in the tissues was calculated according to the following formula: Brain water $\%=[($ wet weight-dry weight)/wet weight $] \times 100$.

Morris water maze test. The spatial learning and memory of the rats was evaluated by Morris water maze, as described previously, 7-10 days after ICH (28). A total of 20 rats were used for this test (10 per group). Each rat was allowed to find the submerged platform within $60 \mathrm{sec}$. A total of 4 trials were conducted on the first testing day (7 days post-ICH), which was considered as a training procedure. Formal testing was then conducted daily on days 8,9 and 10 . At $5 \mathrm{~h}$ after the final testing on day 10 , the platform was removed from the tank and a probe trial was performed to assess the learning and memory performance of each rat.

$R T$-PCR . Semi-quantitative PCR was conducted at 4 days post-ICH. Tissues were homogenized on ice with TRIzol reagent (Invitrogen; Thermo Fisher Scientific, Inc.) and total RNA was extracted, according to the manufacturer's protocol. High-quality RNA was reverse transcribed into cDNA with a Reverse Transcription kit (Takara Biotechnology Co., Ltd., Dalian, China). GAP-43 and GAPDH primers were used. GAP-43 sense: 5'-CATGAGAAGTATGACAACAGCCT-3', GAP-43 antisense 5'-AGTCCTTCCACGATACCAAAGT-3'; GAPDH sense: 5'-CTGCAACCAAAATTCAGGCTA-3', GAPDH antisense 5'-CATCAGCAACGGGAGCAT-3'. The PCR reaction system consisted of cDNA (10 ng), primers $(0.1 \mu \mathrm{M})$, deoxy-ribonucleoside triphosphate $(1 \mathrm{mM})$, Taq DNA polymerase $(5 \mathrm{U}), 10 \mathrm{X}$ buffer $(2.5 \mu \mathrm{l})$, and double distilled water to a final volume of $25 \mu \mathrm{l}$. PCR amplification was performed using SYBR Premix Ex Taq (Takara Biotechnology Co., Ltd.) on an ABI StepOnePlus Real-time PCR system (Thermo Fisher Scientific, Inc.). The PCR protocol was as follows: $95^{\circ} \mathrm{C}$ for $2 \mathrm{~min}, 40$ cycles of $95^{\circ} \mathrm{C}$ for $10 \mathrm{sec}$ and $60^{\circ} \mathrm{C}$ for $40 \mathrm{sec}$. For semi-quantitative PCR, products were analyzed using agarose gel electrophoresis and the corresponding optical density ratio was quantified (optical density value of the specific gene/optical density value of GAPDH).

Western blot analysis. Western blotting was conducted at 4 days post-ICH. Rat perihematomal tissues were lysed in Tissue Protein Lysis Solution (Thermo Fisher Scientific, Inc.) supplemented with 5\% Proteinase Inhibitor Cocktail (Sigma-Aldrich; Merck KGaA), incubated on ice for $30 \mathrm{~min}$, and centrifuged at $15,000 \times g$ at $22-24^{\circ} \mathrm{C}$ for $15 \mathrm{~min}$. Protein concentrations were determined using bicinchoninic acid protein assay reagents (Nanjing Jiancheng Bioengineering Institute, Nanjing, China). Samples were loaded ( $3 \mu 1$ per line) onto $10 \%$ SDS-PAGE, and transferred to polyvinylidene difluoride membranes for $60 \mathrm{~min}$. Non-specific binding sites were blocked with 5\% bovine serum albumin (Bio-Rad Biotechnology, Inc.) at $22-24^{\circ} \mathrm{C}$ for $1 \mathrm{~h}$, then incubated with various antibodies: Rabbit anti-rat GAP-43 (cat. no. sc10786), IL-1 $\beta$ (cat. no. sc7884), IL-6 (cat. no. sc7920), IL-10 (cat. no. sc984), TNF- $\alpha$ (cat. no. sc8301), ERK (cat. no. sc292838), p-ERK (cat. no. sc101760), p-JNK (cat. no. sc135642) and $\beta$-actin (cat. no. sc130657) polyclonal antibodies (dilution, 1:1,000; Santa Cruz Biotechnology, Inc., Dallas, TX, USA) overnight at $4^{\circ} \mathrm{C}$. The membranes were then incubated with secondary antibodies (cat. no. sc2955, dilution, 1:5,000; Santa 


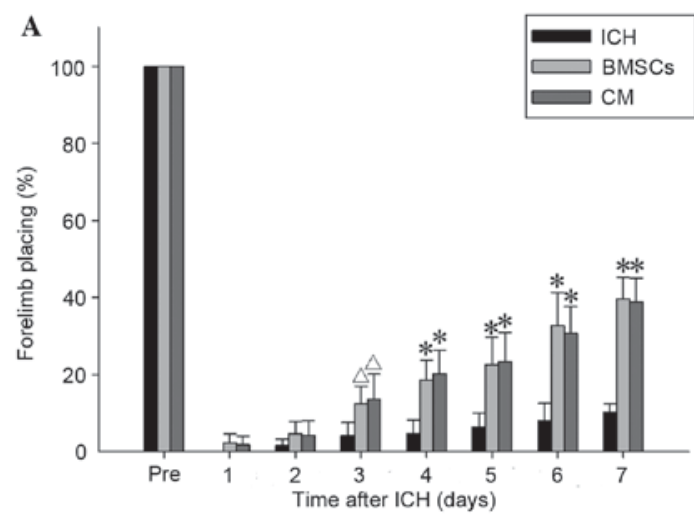

$\mathbf{B}$
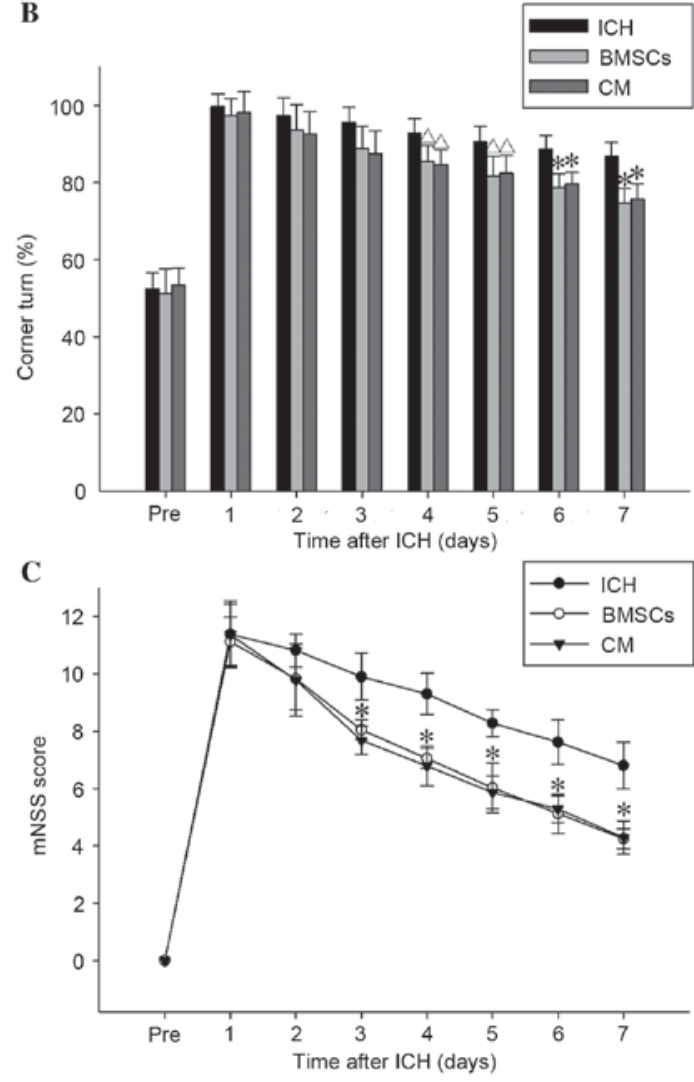

Figure 1. BMSC transplantation and BMSC-CM treatment ameliorated the neurological deficit. Scores of the (A) forelimb-placing test, (B) corner turn test and (C) mNSS evaluation prior to ICH induction (pre), and 1-7 days after induction. Data are presented as the mean \pm standard error ( $\mathrm{n}=5$ per group). ${ }^{*} \mathrm{P}<0.01$ vs. ICH group; ${ }^{\wedge} \mathrm{P}<0.05$ vs. ICH group. BMSC, bone marrow mesenchymal stem cell; CM, conditioned medium; ICH, intracerebral hemorrhage; mNSS, modified neurological severity score.

Cruz Biotechnology, Inc.) for $2 \mathrm{~h}$ at $4^{\circ} \mathrm{C}$. The immunoreactive bands were visualized using an enhanced chemiluminescence reagent (Bio-Rad Laboratories, Inc., Hercules, CA, USA). Blots were scanned by densitometry, and integrated density of pixels was quantified using Image Quant software (version, 5.2; Molecular Dynamics; GE Healthcare Bio-Sciences, Pittsburgh, PA, USA).

Statistical analysis. Data are expressed as the mean \pm standard error. All tests were performed using SPSS software (version 17.0; SPSS, Inc., Chicago, IL, USA). Statistical significance was determined using one-way analysis of variance, and the Student-Newman-Keuls post hoc test was used

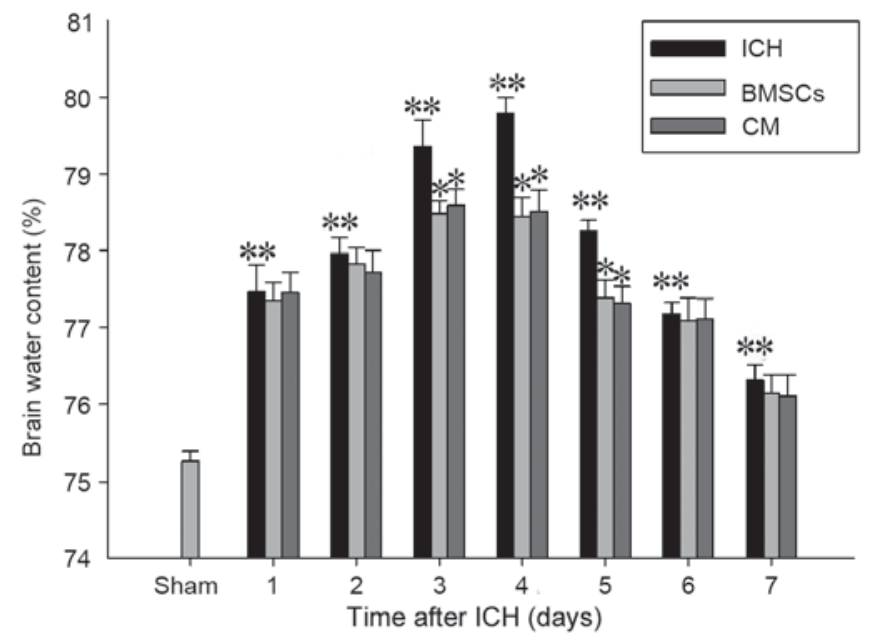

Figure 2. BMSC transplantation and BMSC-CM treatment attenuated brain edema. The time course variation of brain edema was evaluated 1-7 days following induction of $\mathrm{ICH}$. Data are presented as the mean \pm standard error (Sham, 3; ICH, 21; BMSCs, 21; CM, 21). ${ }^{* *} \mathrm{P}<0.01$ vs. Sham group, ${ }^{*} \mathrm{P}<0.01$ vs. ICH group. BMSC, bone marrow mesenchymal stem cell; CM, conditioned medium; $\mathrm{ICH}$, intracerebral hemorrhage.

to determine differences among the groups. $\mathrm{P}<0.05$ was considered to indicate a statistically significant difference.

\section{Results}

Rat mortality. Only two of the total 151 rats succumbed during experimentation. One rat did not survive transplantation of BMSCs during experiment 1 , and the other did not survive treatment with the vehicle in experiment 3 .

Transplantation of BMSCs and BMSC-CM treatment ameliorates neurological deficit. Forelimb-placing and corner turn tests, and the mNSS evaluation, were performed prior to and 1-7 days after ICH. As presented in Fig. 1A, in the ICH model group, there was little recovery of forelimb placing with time. In the BMSC transplantation or BMSC-CM treatment groups, there was a significant recovery compared with in the $\mathrm{ICH}$ model group after 3-7 days $(\mathrm{P}<0.05$ after 3 days; $\mathrm{P}<0.01$ after 4-7 days). As depicted in Fig. 1B, in the corner turn test, the percentage of turns to the right was significantly decreased after 4-7 days in the BMSC transplantation and BMSC-CM treatment groups compared with in the $\mathrm{ICH}$ group $(\mathrm{P}<0.05$ after 4-5 days; $\mathrm{P}<0.01$ after 6-7 days). In addition, Fig. 1C demonstrated that transplantation of BMSCs or treatment with BMSC-CM resulted in significantly improved mNSS scores compared with in the ICH group after 4-7 days $(\mathrm{P}<0.01)$. However, there was no significant difference $(\mathrm{P}>0.05)$ in motor and sensorimotor functions after 1-7 days between the BMSCs and BMSC-CM groups (Fig. 1C).

Transplantation of BMSCs and BMSC-CM treatment attenuates the extent of brain edema. Analysis of brain water content was conducted 1-7 days post-ICH to identify the effects of BMSC transplantation and BMSC-CM on cerebral edema. As demonstrated in Fig. 2, ICH caused a marked increase in brain water content compared with the sham-operated group $(\mathrm{P}<0.01)$. The mean brain water content of rat brains 
A

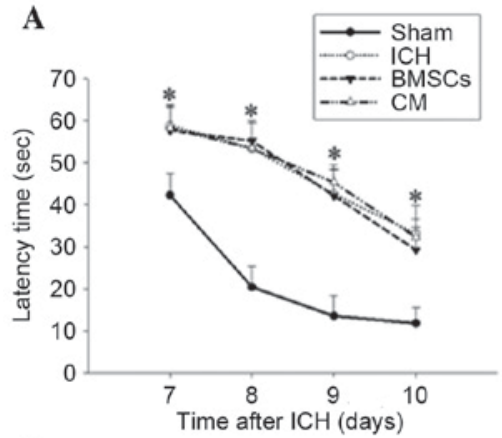

D

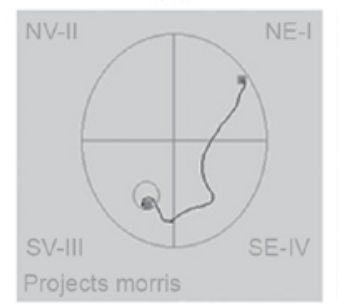

(e)

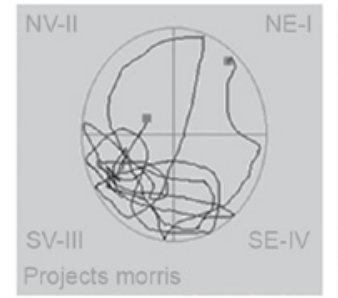

B

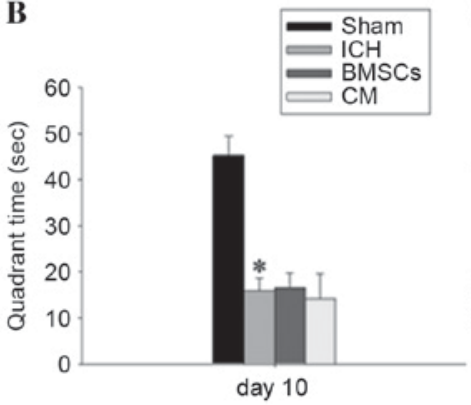

(c)

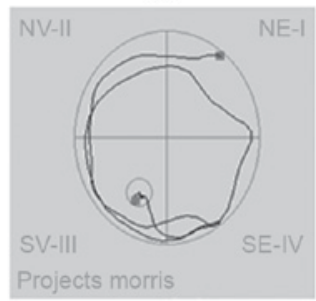

(f)

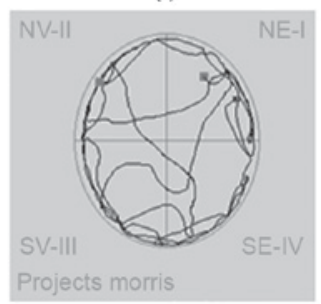

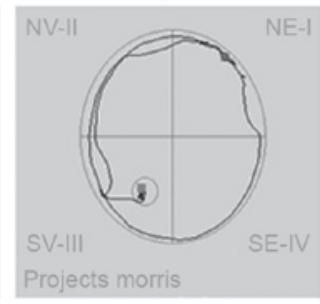

(g)

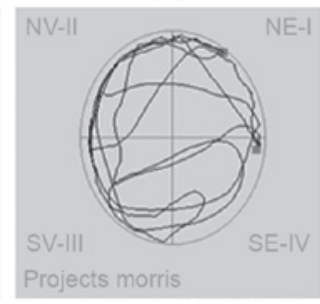

C

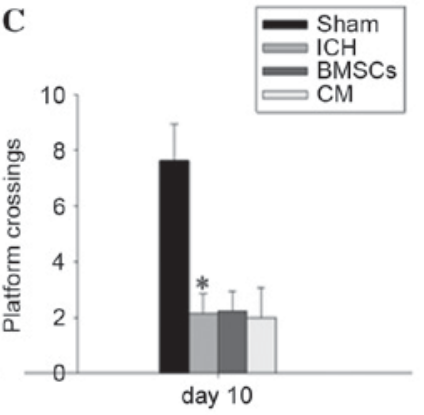

(d)

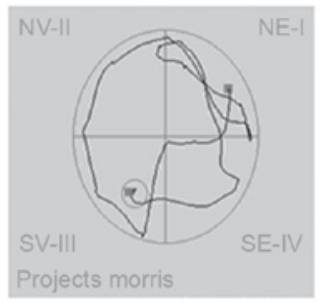

(h)

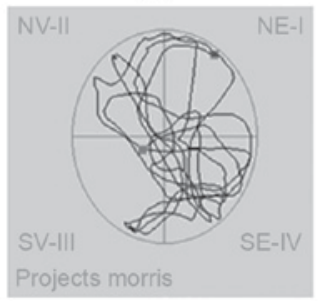

Figure 3. Effects of BMSC transplantation and BMSC-CM treatment on ICH-induced learning and memory dysfunction. (A-C) Latency and probe trial results of Sham, ICH, BMSCs and BMSC-CM-treated animals, as determined by Morris water maze test. (A) Time spent finding the submerged platform, 7-10 days after ICH. (B) Exploration time spent in the quadrant that initially contained the platform, on day 10 . (C) Number of times crossing the platform on day 10 in the probe trial. (D) Representative traces indicating the sample paths of the rats from (a-d) the maze latency trials and (e-h) the probe trials on day 10. Data are presented as the mean \pm standard error ( $\mathrm{n}=5 /$ group). ${ }^{*} \mathrm{P}<0.01$ vs. Sham group. BMSC, bone marrow mesenchymal stem cell; $\mathrm{CM}$, conditioned medium; ICH, intracerebral hemorrhage.

was significantly increased in the ICH group compared with in the BMSCs or BMSC-CM groups after 3-5 days $(\mathrm{P}<0.01)$. However, there was no significant difference $(\mathrm{P}>0.05)$ in brain water content after 1-7 days between the BMSCs and BMSC-CM groups.

Transplantation of BMSCs or BMSC-CM treatment has no effect on ICH-induced cognitive function deficits. To determine whether BMSC transplantation and BMSC-CM treatment led to improved cognitive functional outcome post-ICH, a Morris water maze study was conducted 7-10 days after ICH (Fig. 3). Fig. 3A illustrates the effects of BMSC transplantation and BMSC-CM on latency time for finding the hidden platform during latency trials. Latency time in the ICH groups was progressively longer compared with in the sham group, in a time-dependent manner; however, there was no significant difference between the ICH, BMSCs and BMSC-CM treatment groups. Representative trace diagrams indicating the latency time for finding the submerged platform on day 10 are depicted in Fig. 3Da-d. In probe trials, which are characterized by the removal of the hidden platform (Fig. 3B and C), ICH rats displayed a reduced bias towards the goal quadrant, which previously contained the platform. They spent less time in the goal quadrant and had less platform crossings compared with their sham counterparts. In addition, this phenomenon was not altered in the BMSCs and BMSC-CM groups. Representative traces obtained during the specified probe trials are depicted in Fig. 3De-h.

BMSC-CM treatment reduces the expression of IL-1 $\beta, I L-6$ and TNF- $\alpha$, but not IL-10, in perihematomal tissues. To explore the anti-inflammatory effects of BMSC-CM treatment on ICH, the expression levels of inflammatory cytokines, including TNF- $\alpha$, IL- $1 \beta$ and IL- 6 , and the anti-inflammatory cytokine IL-10, were measured 4 days after ICH in the perihematomal tissues by western blot analysis. As demonstrated in Fig. 4, the protein expression levels of IL-1 $\beta$, IL- 6 and TNF- $\alpha$ were significantly reduced in the brains of BMSC-CM-treated rats compared with in the ICH group (IL- 6 and TNF- $\alpha, \mathrm{P}<0.01$; IL-1 $\beta, \mathrm{P}<0.05)$. Conversely, the expression levels of IL-10 were increased compared with in the ICH group $(\mathrm{P}<0.01)$.

BMSC-CM treatment enhances phosphorylation of ERK1/2 in perihematomal tissues. The present study explored whether BMSC-CM treatment could enhance the activation of cell signaling. As demonstrated in Fig. 5, phosphorylation of ERK1/2 and JNK was significantly elevated in the perihematomal region 4 days after $\mathrm{ICH}(\mathrm{P}<0.01)$, whereas BMSC-CM treatment further enhanced ERK1/2 phosphorylation (Fig. 5B, $\mathrm{P}<0.01)$. However, BMSC-CM treatment had no effect on 
A

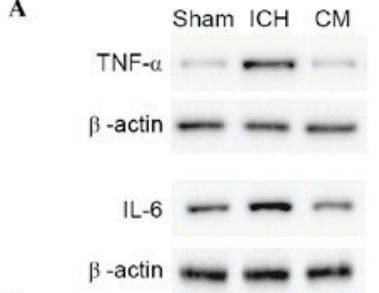

B
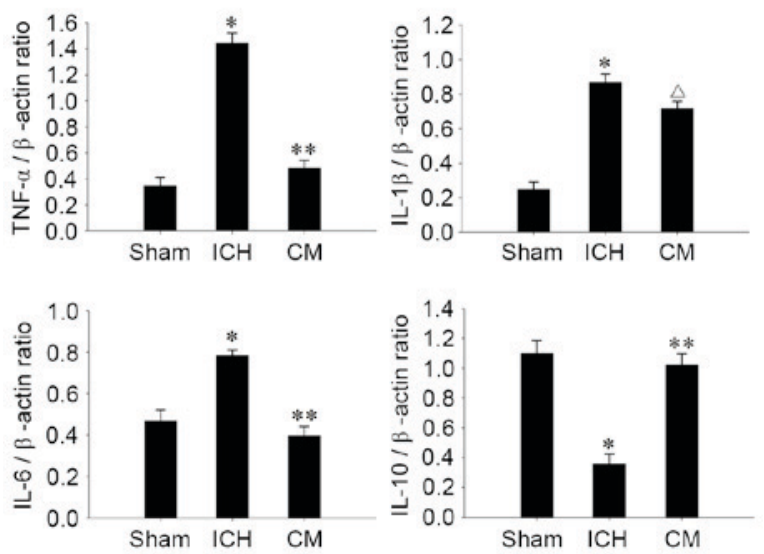

Figure 4. Effects of BMSC-CM treatment on inflammatory cytokine expression. (A) Western blot analysis was used to detect the levels of inflammatory cytokines in the perihematomal tissues of rats 4 days following ICH or sham operation. (B) Semi-quantitative results of inflammatory cytokine expression were expressed as the ratio of the densitometries to $\beta$-actin bands. Data are presented as the mean \pm standard error ( $\mathrm{n}=5 /$ group). ${ }^{*} \mathrm{P}<0.01$ vs. Sham group; ${ }^{* *} \mathrm{P}<0.01$ vs. ICH group; ${ }^{\Delta} \mathrm{P}<0.05$ vs. ICH group. BMSC, bone marrow mesenchymal stem cell; CM, conditioned medium; ICH, intracerebral hemorrhage; IL, interleukin; TNF- $\alpha$, tumor necrosis factor- $\alpha$.

JNK activation in the perihematomal region 4 days after $\mathrm{ICH}$ (Fig. 5C, P>0.05).

BMSC-CM rescues GAP-43 transcription and expression in perihematomal tissues. To determine the effects of BMSC-CM on GAP-43 transcription and expression post-ICH, the expression levels of GAP-43 were detected by RT-PCR and western blot analysis. RT-PCR (Fig. 6A) demonstrated that the mRNA expression levels of GAP-43 in the ICH group were markedly increased 4 days following the operation $(\mathrm{P}<0.01)$. In the $\mathrm{CM}$ group, BMSC-CM treatment significantly enhanced the mRNA expression levels of GAP-43 compared with in the ICH rats $(\mathrm{P}<0.01)$. The results of western blot analysis (Fig. 6B) indicated that ICH induced the elevation of GAP-43 protein compared with the Sham group $(\mathrm{P}<0.01)$, and the expression of GAP-43 was markedly reinforced following BMSC-CM treatment $(\mathrm{P}<0.01)$.

PD98059 reverses the elevation of GAP-43 transcription and expression following BMSC-CM treatment in perihematomal tissues. To further investigate whether the activation of GAP-43 following BMSC-CM treatment was regulated by the ERK1/2 signaling pathway, a p-ERK1/2 inhibitor, PD98059, was intrathecally administered. Fig. 7 depicts the alterations in inflammatory cytokine level, cell signaling, and GAP-43 transcription and expression following the administration of PD98059 4 days after ICH. Notably, PD98059 inhibited the phosphorylation of ERK1/2 (Fig. 7F, P<0.01). The mRNA and protein expression levels of GAP-43 were also significantly decreased in the PD598059 group compared with in the vehicle group (Fig. 7H and $\mathrm{J}, \mathrm{P}<0.01$ ). However, there was no significant difference $(\mathrm{P}>0.05)$ in the expression of inflammatory cytokines between the vehicle and PD98059 treatment groups.

\section{Discussion}

The present study demonstrated that intraparenchymal treatment with BMSC-CM 1 day after ICH exerted neuroprotective effects in rats. Treatment with BMSC-CM attenuated post-ICH cerebral edema after 3-5 days and improved the motor functions of ICH rats after 3-7 days. Furthermore, the current study investigated the mechanisms underlying the protective effects of BMSC-CM on ICH. At the molecular level, the expression levels of GAP-43 and inflammatory cytokines in the perihematomal tissues were detected 4 days after $\mathrm{ICH}$. Treatment with BMSC-CM resulted in a marked elevation in the expression of GAP-43 and IL-10, as well as a significant reduction in the expression levels of IL-1 $\beta$, TNF- $\alpha$ and IL-6. These findings suggested that BMSC-CM acts by inhibiting neuroinflammatory activation, and regulating the expression of GAP-43. Following administration of the p-ERK1/2 inhibitor, PD98059, in BMSC-CM-treated rats, the mRNA and protein expression levels of GAP-43 were significantly decreased, suggesting that the ERK1/2 signaling pathway is essential for GAP-43 expression. A previous study reported that CM from human adipose-derived MSCs mediated protection in neurons following glutamate excitotoxicity by regulating energy metabolism and GAP-43 expression (29). In addition, a recent in vivo study demonstrated that $\mathrm{CM}$ from human umbilical cord MSCs contributed to vascular remodeling in the ischemic brain, which serves an important role in functional outcome in a rat model of stroke (25). These findings, together with the observations of the present study, may aid to improve current understanding regarding stem cell-CM-mediated neuroprotection in neurological disorders.

ICH has a complex pathology with numerous diverse and incompletely clarified etiologies and prognoses. Despite various promising preclinical experiments, including the evaluation of anti-inflammatory, anti-hypertensive and neuroprotective drugs, only symptomatic treatments are currently effective (8). In this context, the search for novel alternatives is a necessity for ICH treatment. A previous study regarding stem cell transplantation in $\mathrm{ICH}$ animal models demonstrated that transplantation attenuated hematoma and improved neurological behaviors (30). Among miscellaneous stem cells, neural stem cells (NSCs) and MSCs are the most widely used and are considered promising in the treatment of $\mathrm{ICH}$. As a result of the distinct capacity of NSCs to differentiate into functional neural cells, the neuroprotective effect may result from transplantation of NSCs replacing damaged neurons. Conversely, the efficacy of MSCs, as demonstrated in models of neurological disease, may be due to the cells' capacity to secrete various neurotrophins, such as brain-derived neurotrophic factor (BDNF), nerve growth factor (NGF) and vascular endothelial growth factor (VEGF), all of which potentially contribute to functional recovery following ICH (31-33). In particular, BMSCs have been regarded as candidates for cell 
A

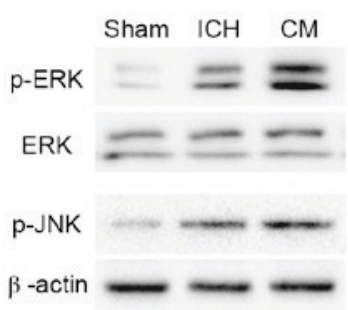

B

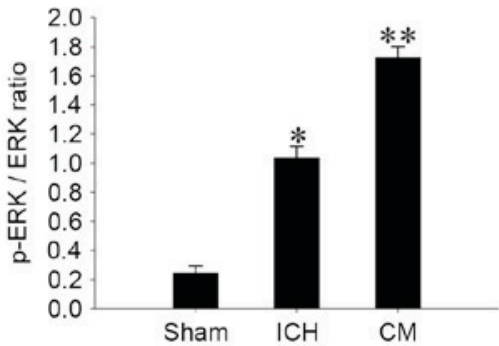

C

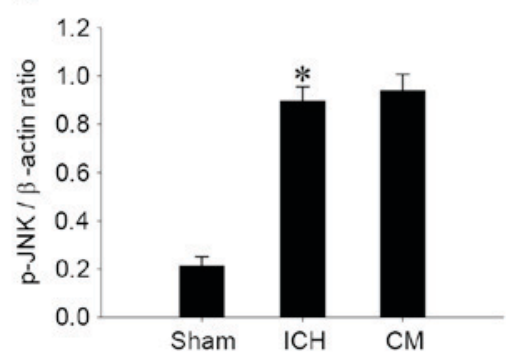

Figure 5. Effects of BMSC-CM treatment on the activation of ERK1/2 and JNK. (A) Western blot analysis detected expression levels of cell signaling proteins in the perihematomal tissues of rats 4 days following ICH or sham operation. (B) Semi-quantitative expression levels of p-ERK1/2 were expressed as the ratio of the densitometries to the ERK1/2 bands. (C) Semi-quantitative results of p-JNK expression were expressed as the ratio of the densitometries to $\beta$-actin bands. Data are presented as the mean \pm standard error ( $\mathrm{n}=5$ /group). $\mathrm{P}<0.01$ vs. Sham group; ${ }^{* *} \mathrm{P}<0.01 \mathrm{vs.} \mathrm{ICH}$ group. BMSC, bone marrow mesenchymal stem cell; CM, conditioned medium; ERK, extracellular signal-regulated kinase; ICH, intracerebral hemorrhage; JNK, c-Jun N-terminal kinase; p-, phosphorylated.
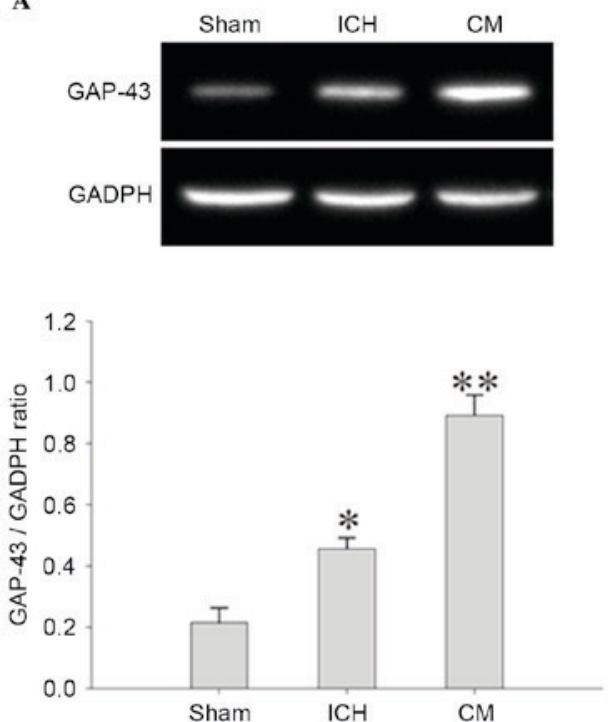
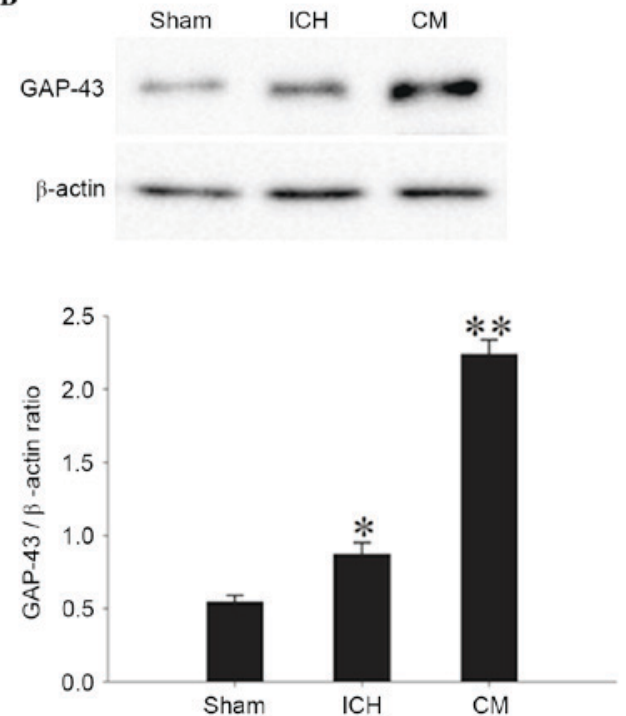

Figure 6. Effects of BMSC-CM treatment on the transcription and expression of GAP-43. (A) Reverse transcription polymerase chain reaction analysis was used to detect the mRNA expression levels of GAP-43 in the perihematomal tissues of rats 4 days after ICH or sham operation (n=5/group). Semi-quantitative results of GAP-43 mRNA expression were expressed as the ratio of densitometries to GADPH bands. (B) Western blot analysis was used to detect the protein expression levels of GAP-43 in the perihematomal tissues of rats 4 days after ICH or sham operation ( $\mathrm{n}=5 / \mathrm{group}$ ). Semi-quantitative results of GAP-43 protein expression were expressed as the ratio of densitometries to $\beta$-actin bands. Data are presented as the mean \pm standard error. ${ }^{*} \mathrm{P}<0.01$ vs. Sham group; ${ }^{* *} \mathrm{P}<0.01$ vs. ICH group. BMSC, bone marrow mesenchymal stem cell; CM, conditioned medium; ICH, intracerebral hemorrhage; GAP-43, growth-associated protein-43.

therapy as they can express an extensive ability for expansion in vitro and can be readily procured by a simple bone marrow aspiration (34). The present study indicated that the neuroprotective effects of BMSC implantation and BMSC-CM treatment have no significant difference to each other and both prevent post-ICH cerebral edema and improve neurological function. Therefore, the observed beneficial effects of BMSCs are mediated by soluble factors secreted by these BMSCs into CM.

The present results also suggested that BMSC-CM was able to exert anti-inflammatory effects in perihematomal tissues of ICH rats. Previous studies have focused on therapies for secondary brain injury following $\mathrm{ICH}$ and indicated that inflammatory events within the first 7 days are the crucial contributor to secondary brain injury $(20,35)$. These findings indicated that attenuation of inflammatory factors may improve neurological recovery following $\mathrm{ICH}$. It has been demonstrated that inflammatory cytokines, including TNF- $\alpha$,
IL-1 $\beta$ and IL-6, produce cerebral edema and brain damage post-ICH by recruiting lymphocyte and neutrophil infiltration into perihematomal tissues $(20,36,37)$. Conversely, the anti-inflammatory cytokine IL-10 exerts beneficial effects against brain damages post-ICH (38). A previous study indicated that compared with the pathophysiology of traumatic brain injury and cerebral ischemia, the pathophysiology of ICH was not associated with the expression of TNF- $\alpha$, IL- $1 \beta$ and IL- 6 in the acute phase ( $1 \mathrm{~h}$ after ICH) in the perihematomal region (35). In the present study, 4 days after ICH, TNF- $\alpha$, IL-1 $\beta$ and IL-6 levels were decreased, whereas IL-10 expression was significantly elevated in the perihematomal tissues of BMSC-CM-treated rats. These results provided evidence to suggest that BMSC-CM is able to effectively regulate the production and secretion of inflammatory cytokines in the perihematomal regions of the ICH-injured brain.

The present study focused on the association between ERK1/2 signaling and GAP-43 transcription and expression. 

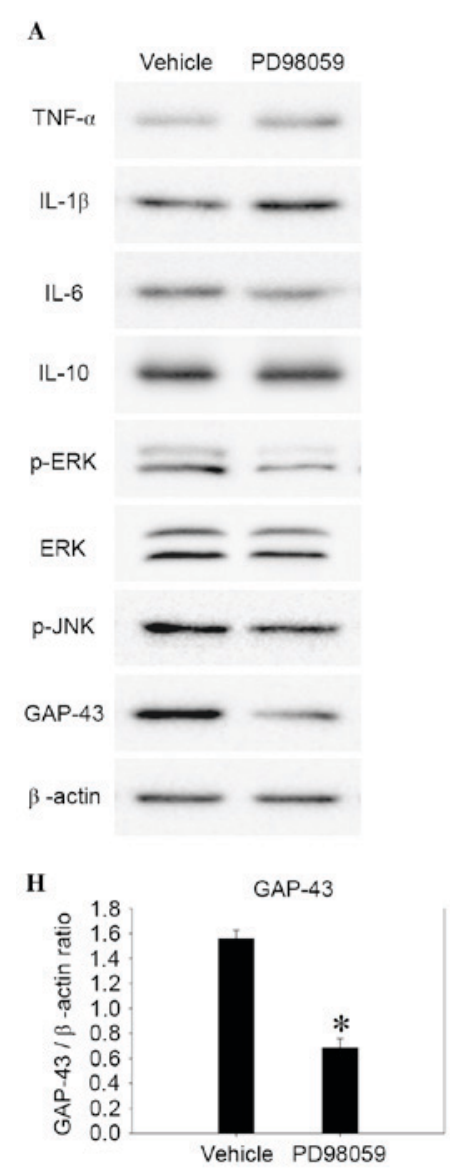
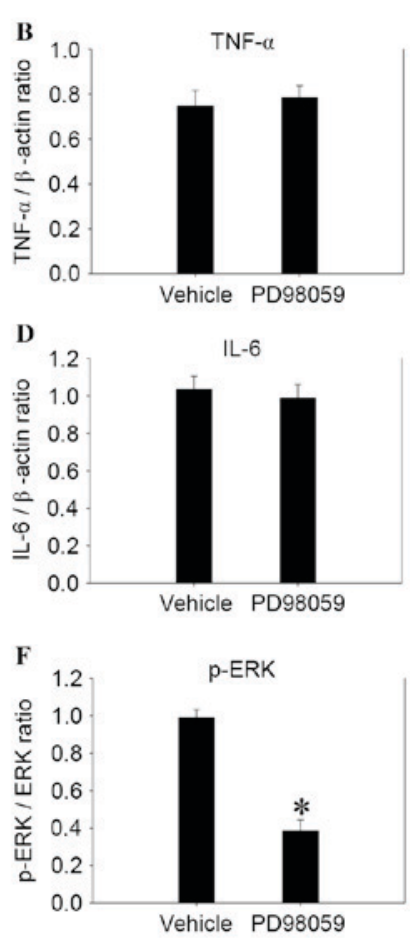

I

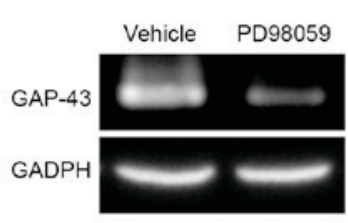

C

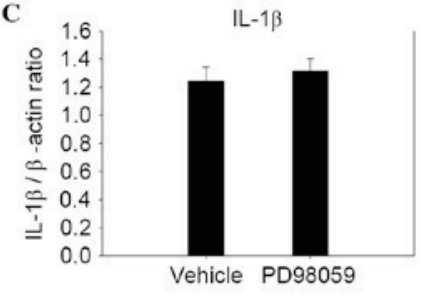

E
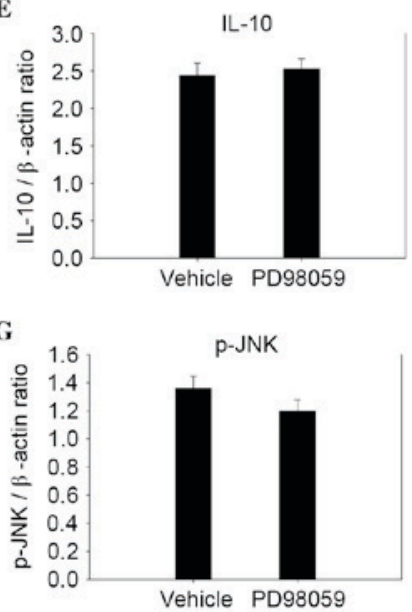

$\mathbf{J}$

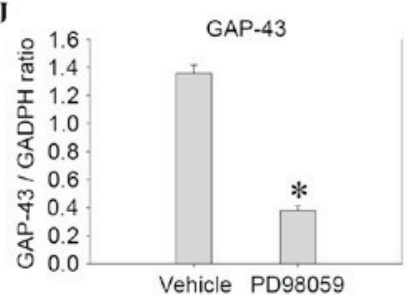

Figure 7. Effects of PD98059 on BMSC-CM-treated rats. (A) Western blot analysis was used to detect the expression levels of inflammatory cytokines, cell signaling proteins and GAP-43 in the perihematomal tissues of rats 4 days following treatment with the vehicle (5\% dimethyl sulfoxide) or p-ERK1/2 inhibitor (PD98059). (B-H) Semi-quantitative results of protein expression are presented as the mean \pm standard error ( $\mathrm{n}=5 /$ group). (I) Reverse transcription polymerase chain reaction analysis was used to detect the mRNA expression levels of GAP-43, following vehicle or PD98059 treatment. (J) Semi-quantitative results of GAP-43 mRNA expression were expressed as the ratio of densitometries to GADPH bands. Data are presented as the mean \pm standard error (n=5/group).

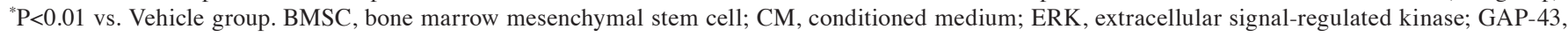
growth-associated protein-43; IL, interleukin; JNK, c-Jun N-terminal kinase; TNF- $\alpha$, tumor necrosis factor- $\alpha$.

It appears that the phosphorylation of ERK1/2 is a major mechanism in BMSC-CM-induced GAP-43 expression in ICH rats. A previous study suggested that GAP-43 was required for BDNF-induced ERK1/2 activation (39). The findings of the present study, together with previous hypotheses, highlight the possibility that heterogeneous factors, including BDNF, NGF and VEGF, are involved in a similar signaling pathway leading to ERK1/2 phosphorylation (40). To the best of our knowledge, the present study is the first to suggest that the ERK1/2-GAP-43 signaling pathway may be considered a potential novel application target of BMSC-CM for the treatment of CNS diseases.

In addition to motor deficits post-ICH, learning and memory impairments may evolve that also adversely impair lifestyle (41). Previous studies have confirmed the role of the striatum in cognition, and extravasation of blood into the caudate putamen of rats produces a learning and memory impairment $(42,43)$. In the present study, the rat cognitive test was conducted 7-10 days after ICH. BMSC transplantation and BMSC-CM treatment produced no significant effect on the learning and memory functions of ICH rats. However, it remains uncertain as to whether BMSC or BMSC-CM treatment has beneficial effects on learning and memory functions in the ICH model used in the present study, since the study was conducted during a relatively short time period. In addition, the majority of patients with ICH are elderly; however, the rats used in the present study were selected from a fixed age group with a high proportion of young rats. Therefore, future studies are required to explore the long-term effects of BMSC-CM-mediated neuroprotection in aged animal models.

In conclusion, treatment with BMSC-CM may be considered an alternative to direct BMSC transplantation in a rat model of ICH. The mechanism underlying BMSC-CM-mediated neuroprotection may involve anti-inflammatory effects, and activation of GAP-43 transcription and expression via ERK1/2 phosphorylation.

\section{Acknowledgements}

The present study was supported by a grant from the Natural Science Foundation of Hebei Province (grant no. H2014105079). 


\section{References}

1. Tow fighi A and Saver JL: Stroke declines from third to fourth leading cause of death in the United States: Historical perspective and challenges ahead. Stroke 42: 2351-2355, 2011.

2. Qureshi AI, Mendelow AD and Hanley DF: Intracerebral haemorrhage. Lancet 373: 1632-1644, 2009.

3. Cheon SH: The effect of a skilled reaching task on hippocampal plasticity after intracerebral hemorrhage in adult rats. J Phys Ther Sci 27: 131-133, 2015.

4. Lok J, Leung W, Murphy S, Butler W, Noviski N and Lo EH: Intracranial hemorrhage: Mechanisms of secondary brain injury Acta Neurochir Suppl 111: 63-69, 2011.

5. Wasserman J and Schlichter L: Neuron death and inflammation in a rat model of intracerebral hemorrhage: Effects of delayed minocycline treatment. Brain Res 1136: 208-218, 2007.

6. Kidwell CS, Latour LL, Hsia AW, Merino JC, Burgess RE, Copenhaver BR, Castle A and Warach S: Demonstration of blood-brain barrier disruption in humans with primary intracerebral hemorrhage. Stroke 38: 464, 2007.

7. Xi G, Keep RF and Hoff JT: Erythrocytes and delayed brain edema formation following intracerebral hemorrhage in rats. J Neurosurg 89: 991-996, 1998.

8. Katsuki H: Exploring neuroprotective drug therapies for intracerebral hemorrhage. J Pharmacol Sci 114: 366-378, 2010.

9. Meiri KF, Pfenninger KH and Willard MB: Growth-associated protein, GAP-43, a polypeptide that is induced when neurons extend axons, is a component of growth cones and corresponds to pp46, a major polypeptide of a subcellular fraction enriched in growth cones. Proc Natl Acad Sci USA 83: 3537-3541, 1986.

10. Maier DL, Mani S, Donovan SL, Soppet D, Tessarollo L, McCasland JS and Meiri KF: Disrupted cortical map and absence of cortical barrels in growth-associated protein (GAP)-43 knockout mice. Proc Natl Acad Sci USA 96: 9397-9402, 1999.

11. Allegra Mascaro AL, Cesare P, Sacconi L, Grasselli G, Mandolesi G, Maco B, Knott GW, Huang L, De Paola V and Pavone FS: In vivo single branch axotomy induces GAP-43-dependent sprouting and synaptic remodeling in cerebellar cortex. Proc Natl Acad Sci USA 110: 10824-10829, 2013.

12. Carmichael ST, Archibeque I, Luke L, Nolan T, Momiy J and Li S: Growth-associated gene expression after stroke: Evidence for a growth-promoting region in peri-infarct cortex. Exp Neurol 193: 291-311, 2005.

13. Stroemer RP, Kent TA and Hulsebosch CE: Acute increase in expression of growth associated protein GAP-43 following cortical ischemia in rat. Neurosci Lett 162: 51-54, 1993.

14. Gorup D, Bohaček I, Miličević T, Pochet R, Mitrečić D, Križ J and Gajović S: Increased expression and colocalization of GAP43 and CASP3 after brain ischemic lesion in mouse. Neurosci Lett 597: 176-182, 2015

15. Ashton BA, Allen TD, Howlett CR, Eaglesom CC, Hattori A and Owen M: Formation of bone and cartilage by marrow stromal cells in diffusion chambers in Vivo. Clin Orthop Relat Res 151: 294-307, 1980.

16. Pereira RF, Halford KW, O'Hara MD, Leeper DB, Sokolov BP, Pollard MD, Bagasra O and Prockop DJ: Cultured adherent cells from marrow can serve as long-lasting precursor cells for bone, cartilage, and lung in irradiated mice. Proc Natl Acad Sci USA 92: 4857-4861, 1995.

17. Chen J, Li Y, Wang L, Zhang Z, Lu D, Lu M and Chopp M: Therapeutic benefit of intravenous administration of bone marrow stromal cells after cerebral ischemia in rats. Stroke 32: 1005-1011, 2001.

18. Caplan AI and Dennis JE: Mesenchymal stem cells as trophic mediators. J Cell Biochem 98: 1076-1084, 2006.

19. Majumdar MK, Thiede MA, Haynesworth SE, Bruder SP and Gerson SL: Human marrow-derived mesenchymal stem cells (MSCs) express hematopoietic cytokines and support long-term hematopoiesis when differentiated toward stromal and osteogenic lineages. J Hematother Stem Cell Res 9: 841-848, 2000.

20. Lee ST, Chu K, Jung KH, Kim SJ, Kim DH, Kang KM, Hong NH, Kim JH, Ban JJ, Park HK, et al: Anti-inflammatory mechanism of intravascular neural stem cell transplantation in haemorrhagic stroke. Brain 131: 616-629, 2008.

21. Daan VP, Biju P, Cho CH, Berthiaume F, Nahmias Y, Tilles AW and Yarmush ML: Mesenchymal stem cell-derived molecules directly modulate hepatocellular death and regeneration in vitro and in vivo. Hepatology 47: 1634-1643, 2008

22. Meirelles LdaS,Fontes AM, Covas DT and Caplan AI: Mechanisms involved in the therapeutic properties of mesenchymal stem cells. Cytokine Growth Factor Rev 20: 419-427, 2009.
23. Chen L, Tredget EE, Wu PY and Wu Y: Paracrine factors of mesenchymal stem cells recruit macrophages and endothelial lineage cells and enhance wound healing. PLoS One 3: e1886, 2008.

24. Li X, Luo Q, Sun J and Song G: Conditioned medium from mesenchymal stem cells enhances the migration of hepatoma cells through CXCR4 up-regulation and F-actin remodeling. Biotechnol Lett 37: 511-521, 2015.

25. Zhao Q, Hu J, Xiang J, Gu Y, Jin P, Hua F, Zhang Z, Liu Y, Zan K, Zhang Z, et al: Intranasal administration of human umbilical cord mesenchymal stem cells-conditioned medium enhances vascular remodeling after stroke. Brain Res 1624: 489-496, 2015.

26. Teng W, Wang L, Xue W and Guan C: Activation of TLR4-mediated NFkappaB signaling in hemorrhagic brain in rats. Mediators Inflamm 2009: 473276, 2009.

27. Cui C, Cui Y, Gao J, Sun L, Wang Y, Wang K, Li R, Tian Y, Song $\mathrm{S}$ and Cui J: Neuroprotective effect of ceftriaxone in a rat model of traumatic brain injury. Neurol Sci 35: 695-700, 2014.

28. Raz L, Zhang QG, Zhou CF, Han D, Gulati P, Yang LC, Yang F, Wang RM and Brann DW: Role of Racl GTPase in NADPH oxidase activation and cognitive impairment following cerebral ischemia in the rat. PLoS One 5: e12606, 2010.

29. Peng H, Liang Z, Piao H, Ji X, Wang Y, Liu Y, Liu R and Liu J: Conditioned medium of human adipose-derived mesenchymal stem cells mediates protection in neurons following glutamate excitotoxicity by regulating energy metabolism and GAP-43 expression. Metab Brain Dis 29: 193-205, 2014.

30. Hu Y, Liu N, Zhang P, Pan C, Zhang Y, Tang Y, Deng H, Aimaiti M, Zhang Y, Zhou H, et al: Preclinical studies of stem cell transplantation in intracerebral hemorrhage: A systemic review and meta-analysis. Mol Neurobiol 53: 5269-5277, 2016.

31. Seyfried D, Ding J, Han Y,Li Y, Chen J and Chopp M: Effects of intravenous administration of human bone marrow stromal cells after intracerebral hemorrhage in rats. J Neurosurg 104: 313-318, 2006.

32. Li Y, Chen J, Chen XG, Wang L, Gautam SC, Xu YX, Katakowski M, Zhang LJ, Lu M, Janakiraman N and Chopp M: Human marrow stromal cell therapy for stroke in rat: Neurotrophins and functional recovery. Neurology 59: 514-523, 2002.

33. Gerdoni E, Gallo B, Casazza S, Musio S, Bonanni I, Pedemonte E, Mantegazza R, Frassoni F, Mancardi G, Pedotti R and Uccelli A: Mesenchymal stem cells effectively modulate pathogenic immune response in experimental autoimmune encephalomyelitis. Ann Neurol 61: 219-227, 2007.

34. Yan C, Lian X, Dai Y, Wang X, Qu P, White A, Qin Y and Du H: Gene delivery by the hSP-B promoter to lung alveolar type II epithelial cells in LAL-knockout mice through bone marrow mesenchymal stem cells. Gene Ther 14: 1461-1470, 2007.

35. Lee IN, Cheng WC, Chung CY, Lee MH, Lin MH, Kuo CH, Weng HH and Yang JT: Dexamethasone reduces brain cell apoptosis and inhibits inflammatory response in rats with intracerebral hemorrhage. J Neurosci Res 93: 178-188, 2015.

36. Ziai WC: Hematology and inflammatory signaling of intracerebral hemorrhage. Stroke 44 (6 Suppl 1): S74-S78, 2013.

37. Aronowski J and Zhao X: Molecular pathophysiology of cerebral hemorrhage: Secondary brain injury. Stroke 42: 1781-1786, 2011.

38. Jiang Y, Wei N, Lu T, Zhu J, Xu G and Liu X: Intranasal brain-derived neurotrophic factor protects brain from ischemic insult via modulating local inflammation in rats. Neuroscience 172: 398-405, 2011.

39. Gupta SK, Mishra R, Kusum S, Spedding M, Meiri KF, Gressens P and Mani S: GAP-43 is essential for the neurotrophic effects of BDNF and positive AMPA receptor modulator S18986. Cell Death Differ 16: 624-637, 2009.

40. Matsuoka Y and Yang J: Selective inhibition of ERK1/2 blocks NGF to BDNF signaling and suppresses the development of and reverses already established pain behavior in rats. Neuroscience 206: 224-236, 2012.

41. Nys GM, van Zandvoort MJ, de Kort PL, Jansen BP, de Haan EH and Kappelle LJ: Cognitive disorders in acute stroke: Prevalence and clinical determinants. Cerebrovasc Dis 23: 408-416, 2007.

42. Lekic T, Hartman R, Rojas H, Manaenko A, Chen W, Ayer R, Tang J and Zhang JH: Protective effect of melatonin upon neuropathology, striatal function, and memory ability after intracerebral hemorrhage in rats. J Neurotrauma 27: 627-637, 2010.

43. Ragozzino ME: The contribution of the medial prefrontal cortex, orbitofrontal cortex and dorsomedial striatum to behavioral flexibility. Ann N Y Acad Sci 1121: 355-375, 2007. 